\title{
Calidad y desarrollo profesional del profesorado universitario, desde la perspectiva de las comunidades de aprendizaje
}

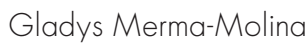

Diego Gavilán-Martín

Recibido: 19/03/2014 - Aceptado: 04/04/2014

\section{Resumen}

En esta investigación, reflexionamos en torno a las competencias docentes que debe tener el profesor universitario desde la perspectiva de las comunidades de aprendizaje, en cada una de las etapas de la gestión del proceso de enseñanzaaprendizaje: 1) planificación y diseño de la docencia, donde se plantean competencias y objetivos, se organizan los contenidos, se eligen los materiales y recursos, y se formula la evaluación; 2) la puesta en acción de lo planificado, previamente (metodología y el uso de estrategias pedagógicas como la motivación y la tutorización); el uso adecuado de medios y materiales; la evaluación, que promueva la reflexión y desarrolle la capacidad crítica; y 3) desarrollo profesional (formación continua), que le proporcione al docente un dominio de la disciplina científica que imparte, y que le posibilite integrar las tecnologías de la información y de la comunicación en la docencia.

\section{Palabras clave}

Competencias docentes, Espacio Europeo de Educación Superior, gestión de la enseñanza, docencia.

\begin{abstract}
In this research, we reflect about the teaching skills that a university profesor should have from the perspective of learning communities at each stage of the management process of teaching and learning: 1) planning and design of teaching where competencies and objectives are set, contents are organized, materials and resources are chosen, and the assessment is made, 2) the implementation of the planned action (methodology and use of teaching strategies such as motivation and tutoring), the appropriate use of media and materials, evaluation, to promote and develop the critical thinking ability, and 3) professional development (continuing education), to provide teachers a domain of scientific discipline to integrate information technology and communication in teaching.
\end{abstract}

\section{Keywords}

Teaching skills, European Higher Education Area, teaching management, teaching.

Gladys Merma Molina es doctora por la Universidad de Alicante. Es profesora ayudante doctora en el Departamento de Didáctica General y Didácticas Específicas de la misma Universidad. Además de los estudios de doctorado, ha realizado un Máster en Comunicación y Cultura, un Máster en Educación y Tecnologías de la Información y de la Comunicación, un Máster en Administración, y un curso de especialista en Dirección de Organizaciones Públicas. Participa en diversos proyectos de investigación. Entre ellos, destacan el Proyecto de Redes de Investigación en Docencia Universitaria, El Proyecto de Pedagogía GITE, Grupo de Innovación Tecnológico-Educativa de la Universidad de Alicante, Proyecto Diseño y Atención a las Oportunidades de Género en la Educación Superior y el Proyecto Capacitación para el Desarrollo con Equidad de Género para Regidoras y Líderes Peruanas. Ha escrito libros y diversos artículos, dentro del ámbito de 
la Educación y de la Filología. Ha coordinado la publicación de diferentes volúmenes. Ha participado en la revisión y publicación de diversos volúmenes, editados por el Instituto de Ciencias de la Educación y por la Facultad de Educación, de la Universidad de Alicante. Ha presentado comunicaciones en congresos nacionales e internacionales. gladys.merma@ua.es

Diego Gavilán Martín es profesor colaborador de la Facultad de Educación de la Universidad de Alicante. Ha realizado el Máster en Profesorado de Educación Secundaria. Actualmente imparte asignaturas del Área de Teoría e Historia de la Educación y otras vinculadas con el uso de la TIC en la educación (Aulas Digitales, Innovación en la Informática). Ha diseñado y creado el sitio Web del Grupo Interdisciplinar de Docencia Universitaria (GIDU-2008) y ha desarrollado el sitio Web del Proyecto Diseño y Atención a las Oportunidades de Género en la Educación Superior (2012), de la Universidad de Alicante. Es un profesional del campo de la comunicación digital y corporativa y el diseño gráfico, web y multimedia. Es fundador del Estudio de Diseño y Publicidad TAIMARA. diego.gavilan@ua.es

\section{Introducción}

La adecuada planificación docente, en el Espacio Europeo de Educación Superior, es una de las cuestiones clave para desarrollar, de manera óptima, la docencia. La capacidad para diseñar y planificar la tarea docente ha sido destacada por muchos autores, como Zabalza (2003). La planificación del proceso de enseñanza-aprendizaje ha de ser el primer paso que oriente nuestra práctica docente profesional. La tarea del profesor abarca distintos ámbitos de actuación, desde priorizar los contenidos más significativos, la comprensión, conseguir la atención de todos los alumnos, el estilo de impartir su docencia, la constante adecuación en función del grupo y la importancia de la participación de los alumnos en todo el proceso educativo.

El docente debe saber unificar conocimientos, utilizar técnicas grupales para comunicar los mensajes y motivar a los alumnos (Sarramona, 1991) porque la formación de los alumnos implica transmisión de un saber (Ios conocimientos), de un saber hacer (las competencias) y de un saber estar (los comportamientos).

Toda actividad exige la presencia de un número variado de competencias, para ser realizada con calidad. La competencia ha sido definida como un conjunto dinámico de atributos, vinculados con el conocimiento y su puesta en práctica, que describen los objetivos de aprendizaje de un programa educativo y cómo los estudiantes actuarán al final de dicho proceso (González y Wagenaar, 2002). Hablar de las competencias que un docente debe desarrollar ya no es un tema novedoso; sin embargo, aún nos falta saber plantear y seleccionar aquellas que nos ayuden a orientar nuestra función hacia la formación integral del alumnado. Asimismo, la utilización de las TIC como herramienta de trabajo innovador, creativo y motivador, junto a la potencialidad de comunicación, son otras de las capacidades que todo docente debe cultivar de forma permanente. La investigación y búsqueda de la información, previa a la evaluación posterior, tanto del conocimiento adquirido por el alumnado como del proceso realizado, son y deben ser generalmente atribuidas también al profesorado universitario competente, dentro del marco establecido en el Espacio Europeo para la Educación Superior.

En este trabajo, reflexionamos sobre la calidad y sobre la importancia del desarrollo profesional del profesor universitario, en el contexto del Espacio Europeo de Educación Superior, desde el enfoque de las comunidades de aprendizaje. Para ello, destacamos tres momentos importantes de la gestión de la enseñanza-aprendizaje: 1) planificación y sistematización — diseño de la guía docente-, en el que se plantean las competencias, se formalizan los objetivos, se organizan los contenidos, se eligen los materiales y recursos, y se formula la evaluación. 2) la puesta en acción de lo planificado, previamente, y 3) la formación continua, que le proporcione al docente un dominio de la disciplina científica que imparte, y que le posibilite integrar las TIC en el desarrollo de las distintas actividades.

\section{Las comunidades de aprendizaje en el desarrollo de competencias docentes de diseño, ejecución de la planificación y desarrollo profesional}

Las comunidades de aprendizaje, desarrolladas por Lave y Wenger (1991), favorecen una actuación pedagógica del docente más innovadora y creativa (O'Sullivan \& Deglau 2006), ya que permiten una mejor integración curricular (Loepp, 1999) y potencia la interconexión entre la teoría y la práctica (Buysse, Sparkman \& Wesley, 2003). Los profesores aprenden a partir de la reflexión compartida con sus compañeros, y los alumnos pueden enfrentar de mejor manera los desafíos que les plantea el aprendizaje en su día a día. Bajo este marco, desarrollamos las tres etapas de gestión de la docencia universitaria: la planificación y diseño de la docencia, la puesta en acción de lo planificado y el desarrollo profesional del docente.

\section{Implicaciones de la planificación y diseño de la guía} docente

El proceso de convergencia del Espacio Europeo de Educación Superior nos sitúa ante el reto de planificar, de 
manera coherente, la docencia. Esta planificación de la docencia se realiza mediante guías didácticas, que sirvan de orientación no solo a los profesores, sino también a los estudiantes. La programación de la docencia tiene, desde luego, un importante papel en el desarrollo de una docencia de calidad. Coordinar los programas de las diversas materias con los compañeros, enriquecer informativamente los programas de forma que dejen claro el sentido del trabajo que se debe desarrollar, homogeneizarlos para facilitar su lectura, contrastarlos con otros programas, ponerlos en la web a disposición de los estudiantes, etc., constituyen operaciones de planificación docente, que los profesores debemos realizar, necesariamente, como parte de nuestra actuación docente. Toda esta planificación y sistematización de la acción educativa se debe reflejar en la guía docente.

La guía docente es una herramienta básica del Sistema Europeo de Transferencia de Créditos (ECTS) para alcanzar el objetivo de "promover la cooperación europea en garantía de calidad mediante el desarrollo de metodologías y criterios comparables" (Declaración de Bolonia). Este documento, elaborado por un equipo de profesores, debe informar al alumno que cursa una asignatura o cualquier materia que forme parte de los estudios superiores oficiales, de la planificación detallada de la asignatura, basada en los principios que guían el proceso de Convergencia en la creación de un Espacio Europeo de Educación Superior.

En la guía docente se establecen los contenidos y los objetivos de la asignatura, así como las competencias, que se deben desarrollar. Asimismo, se propone una metodología, unas actividades y unos criterios de evaluación. Además se incluye una bibliografía básica, así como una estimación del tiempo de trabajo del alumno necesario para llevar a cabo las diversas actividades. Una de las diferencias fundamentales entre la planificación tradicional de las asignaturas y la guía docente actual es que la primera estaba enfocada en el contenido (su estructura, distribución, criterios de evaluación, etc.). En cambio, la segunda se basa, por un lado, en el contenido, pero se enfatiza en el trabajo que realiza el estudiante alrededor de ese contenido.

\section{Claves para elaborar la guía docente}

Como ya dijimos anteriormente, la guía docente es un instrumento al servicio del estudiante. Por ello, debe contener los elementos informativos suficientes como para determinar qué es lo que se pretende que aprenda, cómo se va a hacer, bajo qué condiciones y cómo va a ser evaluado el alumno. En este apartado, y en base a la estructura que debe tener la guía, en la universidad, y en concreto, al modelo de guía planteado por Instituto de Ciencias de la Educación de la Universidad de Alicante (España) (2006), señalamos algunas cuestiones clave, que esperamos puedan servir para su mejor comprensión y diseño.

a Identificación o datos informativos, donde hay que incluir datos básicos de la asignatura: nombre, código, créditos ECTS, etc., los relativos al profesor/profesores que imparten la asignatura, y hay que señalar quién es el coordinador de la asignatura. Es importante tener en cuenta el número de créditos ECTS, que nos permitirá determinar el tiempo disponible para el aprendizaje.

b) Contextualización. Implica situar la asignatura en el perfil profesional y en el plan formativo del grado/ postgrado, así como establecer cómo se coordina con el resto de asignaturas. Asimismo, hay que hacer referencia a aspectos relacionados con la contribución de la asignatura al perfil profesional del título y al desarrollo de las competencias específicas y genéricas con las que se relaciona, justificando su inclusión y su relevancia dentro del plan de estudios; el papel de la asignatura dentro del plan de estudios, mencionando básicamente la materia a la que pertenece y sus características, así como las asignaturas con las que guarda relación (previas y posteriores), describiendo brevemente dicha relación y de qué manera la asignatura da continuidad a asignaturas previas o prepara para asignaturas posteriores (ICE, 2006).

c) Competencias. Son datos proporcionados por la universidad, extraídos de las fichas del plan de estudios. Es importante considerar las referencias para determinar las competencias de la asignatura: Real Decreto de directrices propias de la titulación, Proyecto-borrador de Real Decreto de directrices propias de la titulación, Guía docente de la titulación, Libro Blanco de la Titulaciones (ANECA). Otros documentos supletorios en ausencia de todos los anteriores: Declaración de Berlín Proyecto Tuning, Descriptores de Dublín, etc. Aunque no es la única clasificación, se pueden estructurar según su grado de generalidad/especificidad, y hablamos entonces de competencias generales (también llamadas genéricas o transversales) y competencias específicas (figura 1). 
Competencias generales

Competencias específicas
Instrumentales: permiten utilizar el conocimiento como un instrumento o medio, que les permita resolver cualquier situación problemática de la vida cotidiana.

Interpersonales: desarrollo de habilidades comunicativas, favorecen la relación con los demás; facilitan los procesos de interacción social y cooperación.

Sistémicas: permiten percibir y tratar la realidad de modo global.

Conceptuales (Conocimiento teórico): capacidad para conocer y comprender el saber teórico propio de una materia que forme parte del campo disciplinar/ profesional.

Procedimentales: (Conocimiento práctico): permiten desarrollar habilidades vinculadas con el saber hacer. Capacidad para aplicar de modo práctico el conocimiento, en el campo profesional correspondiente.

Actitudinales: actitudes, valores y disposiciones de comportamiento ético que deben estar presentes en el uso del conocimiento y en el ejercicio de las tareas y funciones profesionales, para las que la asignatura está formando.

Figura 1

Competencias generales y específicas d) Objetivos. Se incluirán necesariamente los que constan en la ficha de la asignatura, aunque se pueden añadir más si se estima conveniente, según los bloques temáticos, etc. Podemos clasificar los objetivos como se indica a continuación:

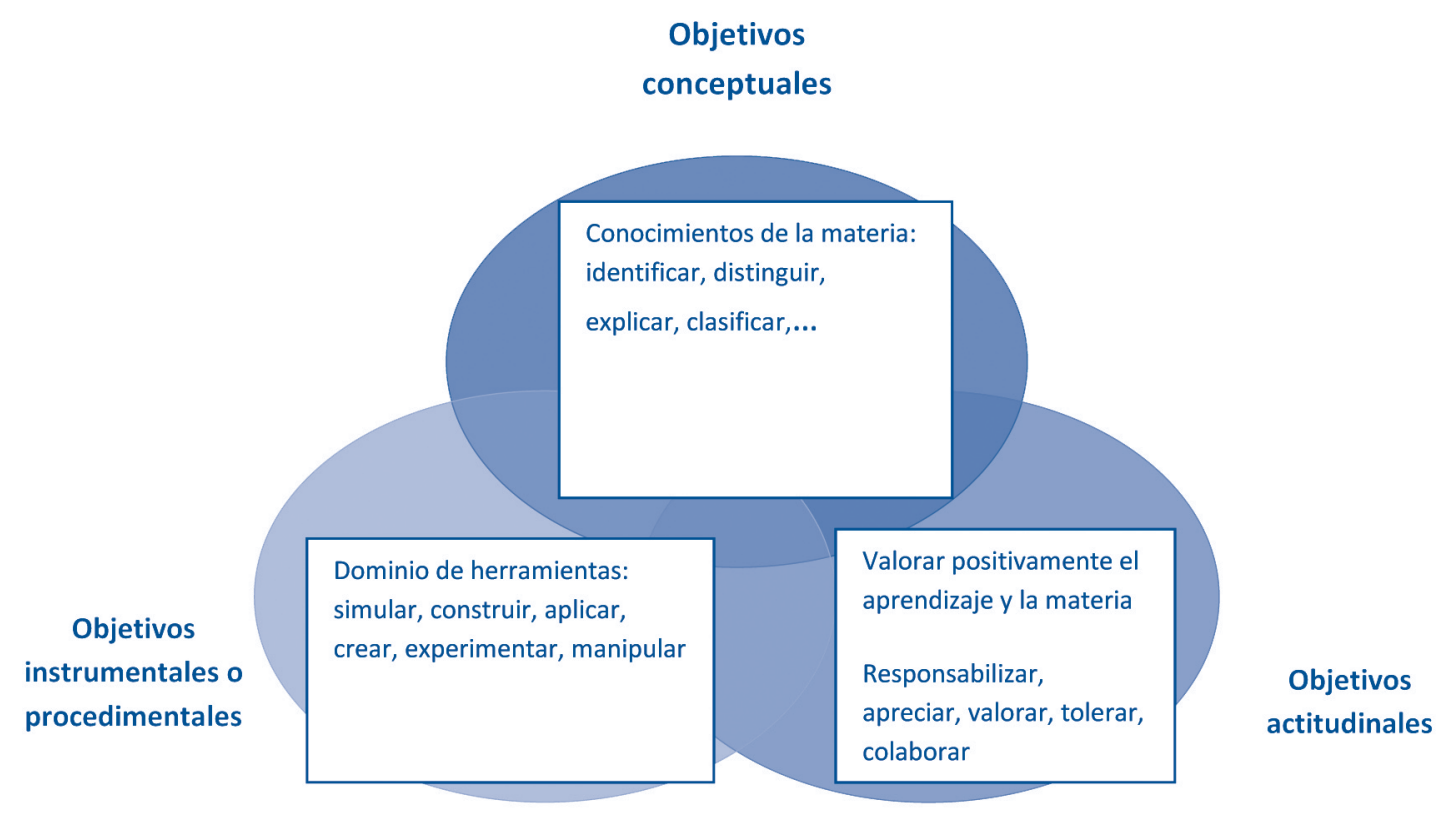

Figura2

Tipología de objetivos 
Los objetivos deben explicitar las mejoras y/o ganancias que los alumnos obtendrán como consecuencia de cursar nuestra asignatura y deben ser claros y concretos.

e) Contenidos. Se recomienda plantear los contenidos por bloques. Debe realizarse una breve descripción de los bloques temáticos, no basta con únicamente con señalarlos.

\section{f) Metodología docente y plan de aprendizaje del alumno}

Metodología docente. El desarrollo y logro de las competencias radica en la metodología y en las estrategias. Por ello, la metodología debe ser variada, coherente con los objetivos de aprendizaje y con los métodos de evaluación y con el diseño de los contenidos.

Plan de aprendizaje del alumno. En este apartado hay que considerar el número de horas presenciales y no presenciales de las diferentes actividades de aprendizaje propuestas, teniendo claro que el número de horas presenciales corresponderá al que aparece en la ficha de la asignatura en el Plan de estudios. Por otro lado, es importante tener en cuenta que las horas totales de presencialidad deben estar comprendidas entre un 30 y un $40 \%$ del total de horas presenciales y no presenciales.

Tabla 1

Metodología y plan de aprendizaje del alumno

METODOLOGÍA DOCENTE Y PLAN DE APRENDIZAJE DEL ALUMNADO

\begin{tabular}{|c|c|c|c|}
\hline ACTIVIDAD DOCENTE $\left({ }^{*}\right)$ & METODOLOGÍA & $\mathrm{HP}\left({ }^{*}\right)$ & HNP \\
\hline \multicolumn{4}{|l|}{ TEORÍA } \\
\hline \multicolumn{4}{|l|}{ SEMINARIOS TEÓRICO-PRÁCTICO } \\
\hline \multicolumn{4}{|l|}{ PRÁCTICAS DE PROBLEMAS } \\
\hline \multicolumn{4}{|l|}{ PRÁCTICAS CON ORDENADOR } \\
\hline \multicolumn{4}{|l|}{ PRÁCTICAS DE CARTOGRAFÍA/MAPAS } \\
\hline \multicolumn{4}{|l|}{ PRÁCTICAS DE LABORATORIO } \\
\hline \multicolumn{4}{|l|}{ PRÁCTICAS-TRABAJOS DE CAMPO } \\
\hline \multicolumn{4}{|l|}{ VISITA A OBRAS, FÁBRICAS... } \\
\hline \multicolumn{4}{|l|}{ PRÁCTICAS CLÍNICAS } \\
\hline \multicolumn{4}{|l|}{ TUTORÍAS GRUPALES } \\
\hline \multicolumn{4}{|l|}{ PRÁCTICAS EN CENTROS SANITARIOS } \\
\hline \multicolumn{4}{|l|}{ PRÁCTICAS EXTERNAS } \\
\hline PRÁCTICAS-TRABAJO DE FIN DE GRADO/MÁSTER & & & \\
\hline$\Lambda$ & - & 25 HORA & \\
\hline
\end{tabular}

HP: número de horas presenciales/curso; HNP: número de horas no presenciales/curso

(*) Datos contemplados en la ficha del Plan de Estudios (Verifica), y, por tanto, fijos.

Fuente: Instituto de Ciencias de la Educación, Universidad de Alicante (España) 
g) Cronograma. Debe ser representativo y orientativo, no solo de una parte, sino de toda la asignatura. Debemos ser capaces de saber regular las tareas, intentando, en lo posible, cumplir el tiempo previsto para cada actividad. El tiempo debe ser gestionado de manera eficaz. Es importante saber calcular el tiempo necesario para realizar cada tarea (tabla 2).

Tabla 2

Temporalización

\begin{tabular}{|c|c|c|c|c|c|}
\hline \multicolumn{2}{|c|}{ ASIGNATURA } & \multicolumn{4}{|c|}{$\begin{array}{l}\text { CRONOGRAMA DE TEMPORALIZACIÓN } \\
\text { SEMANAL DE DEDICACIÓN DEL ESTUDIANTE }\end{array}$} \\
\hline \multirow[b]{2}{*}{ 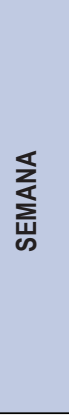 } & \multirow[b]{2}{*}{$\begin{array}{l}\text { UNIDAD } \\
\text { DIDÁCTICA }\end{array}$} & \multicolumn{2}{|c|}{$\begin{array}{l}\text { ACTIVIDADES } \\
\text { PRESENCIALES }\end{array}$} & \multicolumn{2}{|c|}{$\begin{array}{c}\text { ACTIVIDADES } \\
\text { NO PRESENCIALES }\end{array}$} \\
\hline & & $\begin{array}{l}\text { DESCRIPCIÓN } \\
\text { Ejemplos: } \\
\text { clase de teoría, } \\
\text { prácticas de } \\
\text { problemas, } \\
\text { tutorías } \\
\text { grupales, etc. }\end{array}$ & 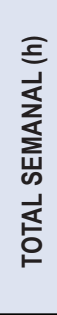 & $\begin{array}{l}\text { DESCRIPCIÓN } \\
\text { Ejemplos: trabajo } \\
\text { individual, trabajo } \\
\text { cooperativo, otras }\end{array}$ & 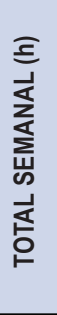 \\
\hline 1 & & & 3 & & 4.5 \\
\hline 2 & & & & & \\
\hline 3 & & & & & \\
\hline 4 & & & & & \\
\hline$\ldots$ & & & & & \\
\hline 15 & & & & & \\
\hline $16-18^{*}$ & & & & & \\
\hline & & & & & \\
\hline & TAL HORAS & DEL ESTUDIAN & ECTS & RAS & \\
\hline
\end{tabular}

* Semanas de evaluación, tras período lectivo.

Fuente: Instituto de Ciencias de la Educación, Universidad de Alicante (España)

h) Bibliografía y recursos. Hay que incluir una bibliografía general de la asignatura, y también bibliografía específica (por temas). La bibliografía básica debe ser accesible a los alumnos. Es importante añadir, en este apartado, páginas web recomendadas, apuntes, colección de problemas, bibliografía de prácticas y material multimedia complementario. Indicar los materiales que se van a emplear en las sesiones de aprendizaje (fotocopias, cuadernos de trabajo,...). Asimismo, se ha de incluir el uso de las TIC, como un recurso para la enseñanza. como bien sabemos, la tecnologías, adecuadamente empleadas, pueden optimizar el aprendizaje (Merma, 2008).

i) Evaluación. Es imprescindible considerar los criterios (y descripción) y su ponderación en la calificación global de la asignatura. Hay que tener en cuenta la normativa universitaria relativa a la evaluación continua. La prueba final su aportación a la calificación no excederá el 50 \% de la nota final. Es importante mantener al alumno constantemente informado sobre los resultados de su evaluación. 
Tabla 3

Criterios de evaluación

\begin{tabular}{|c|c|c|c|}
\hline \multicolumn{2}{|r|}{ Actividad de evaluación* } & Descripción/criterios & Ponderación \\
\hline \multirow{8}{*}{ 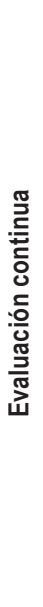 } & Pruebas teórico-prácticas escritas & & \\
\hline & Observaciones del profesor & & \\
\hline & Exposiciones orales & & \\
\hline & Asistencia teoría/prácticas & & \\
\hline & Portafolios o cuaderno de prácticas & & \\
\hline & $\begin{array}{l}\text { Realización de trabajos dirigidos o casos } \\
\text { prácticos }\end{array}$ & & \\
\hline & Pruebas teórico-prácticas & & \\
\hline & Otras & & \\
\hline & Prueba final & & (Max. $50 \%)$ \\
\hline
\end{tabular}

* Se puede descomponer en diferentes actividades individuales o colectivas evaluables (contribuciones en debates, blog, entrega informes, problemas numéricos resueltos, etc.).

Fuente: Universidad de Alicante

\section{La puesta en acción de lo planificado}

\section{El papel del profesor}

Partimos de la preparación psicológica y pedagógica del docente, como animador y guía, de su disposición al diálogo, del empleo de la motivación, la tutorización, y el énfasis, tanto en el trabajo individual como grupal (González, 1997). Una buena motivación implica, entre otras cosas, preparar adecuadamente la primera clase, el comienzo, la acogida, la presentación, dar referencias claras, concisas y concretas. Explicar lo que se pretende, los objetivos, lo que se va a hacer, el programa, la presentación del grupo, y el conocimiento de quiénes lo forman. El profesor siempre debe velar por el desarrollo de un buen ambiente y clima. Debe desarrollar unas buenas habilidades sociales, debe saber establecer relaciones de confianza y de empatía. Aptitudes como la sencillez, la claridad, la imaginación y hacer síntesis frecuentes facilitarán el proceso de enseñanza-aprendizaje.

Para llegar a cabo su labor, el docente tiene a su disposición un cierto número de métodos y de apoyos pedagógicos. Es importante introducir numerosos elementos de motivación, comenzando por mantener un clima favorable en función de las necesidades y de la evolución de grupo. Hay que elegir métodos, ejercicios, que van a permitir al grupo implicarse progresivamente, de manera atractiva. Para ello, partimos de dos premisas: la participación y la corresponsabilidad de cada alumno y del grupo. El docente debe variar sus métodos y no olvidar que el cambio de ritmo es un factor importante para que el grupo trabaje y se sienta bien en el aula.

\section{El desarrollo profesional, una competencia profesional necesaria del docente universitario}

En la actual sociedad del aprendizaje continuo, para sobrevivir, es necesario mantenerse en un proceso de reeducación permanente. Evidentemente, esto obliga al sistema educativo, en general, y al profesorado, en particular, a formarse continuamente (Imbermon, 1993) hasta satisfacer nuevas demandas sociales, definir sus metas en el horizonte de la globalización y rediseñar las competencias profesionales — perfil del docente-.

El profesor universitario tiene un papel sumamente comprometido, en la sociedad moderna (Marina, 2010). Va a formar a los miembros de la sociedad a través de un sistema coactivo amparado por las leyes y teniendo que transmitir unos contenidos en cuya selección no ha tomado parte. Su 
función y competencia tiene la misma complejidad que la del juez. Ambos - juristas y profesores - son elementos que configuran la sociedad. Aunque administrativamente son funcionarios del Estado, el profesor debería ser, sentirse y comportarse como un funcionario de la sociedad. Es posible que las demandas de la sociedad, en materia educativa, o las decisiones políticas, no sean razonables, por lo que el profesor, o algún órgano representativo de la comunidad educativa, debe ejercer un papel crítico, de filtro, de reflexión, de presión en ambos sentidos. Desde esta perspectiva, el profesorado, a través de una formación continua y permanente, ha de concebir su profesión como un proyecto ético, no neutral, donde su finalidad primordial sea formar ciudadanos capacitados laboralmente, pero críticos y creativos. También ha de educar (además de formar) a través de su propia área y/o asignatura, convirtiendo ésta - sea cual sea su base científica-, en una pieza clave dentro de la corresponsabilidad moral. El profesorado ha de formarse para la resolución de conflictos. Pero ello implica un cambio de metodología y adaptación curricular de cada una de las áreas hacia la búsqueda del conocimiento que aminore y resuelva, a su vez, posibles interferencias en el rendimiento académico. Al profesorado no debe bastarle con que el alumnado construya nuevos conocimientos, sino potenciar una situación educativa donde realmente se ejerza una interacción colaborativa entre todos los componentes del sistema. La actividad docente ha de estar abierta y ser completamente plural. El profesorado debe ser un agente pedagógico permanente, y, por tanto, deberá conocer, actualizar e innovar desde el dominio y conocimiento de su disciplina científica para contribuir a una mejor eficiencia del sistema educativo.

\section{Adquisición de competencias básicas en TIC del profesorado universitario}

El profesorado del siglo XXI ha de incorporar en su asignatura diferentes lenguajes icónicos y multimedia para potenciar así los aprendizajes de los estudiantes. Deberá ser capaz, por tanto, de diseñar y preparar materiales didácticos de su asignatura, teniendo en cuenta de manera exhaustiva los mass media. Es por ello que, para integrar y utilizar con eficiencia y eficacia las TIC, el profesorado necesita una buena formación técnica sobre el manejo de estas herramientas tecnológicas y también una formación didáctica que le proporcione un "buen saber hacer pedagógico" con las TIC.

Por las múltiples aplicaciones innovadoras que tiene en todos los ámbitos de nuestra sociedad, el conocimiento y aprovechamiento personal y profesional de los servicios que proporciona internet constituye la parcela más relevante de las competencias en TIC que deben tener los docentes, sin olvidar el resto de las competencias básicas en TIC que necesita todo ciudadano y otras competencias TIC específicas de su campo profesional, muy especialmente la aplicación de estos instrumentos tecnológicos con fines didácticos para facilitar los aprendizajes de los estudiantes. En definitiva, y de acuerdo con diversos estudios realizados al respecto (Cabero y Villar, 1998; Tejada, 1999), podemos enunciar así las competencias didáctico-digitales para los docentes que se recogen de manera más amplia en el siguiente esquema, agrupadas en cuatro dimensiones:

Conocimiento básico de las técnicas e instrumentos tecnológicos, referidas a:

- Sistemas informáticos: gestión básica, virus, archivos,...

- Procesadores de textos: edición, correctores,...

- Navegación en internet: buscar, chatear,...

- Correo electrónico: mensajes, adjuntar documentación,...

- Imagen digital: creación, captura y tratamiento,...

- Webs y presentaciones multimedia.

- Hoja de cálculo y base de datos.

\section{Actualización profesional:}

- Conocer diferentes fuentes de información y programas TIC específicos de su asignatura.

\section{Metodología docente:}

- Integración de recursos TIC en el currículo.

- Aplicación de nuevas estrategias didácticas que aprovechen las TIC.

- Elaboración de documentos y materiales didácticos multimedia.

\section{Actitudes}

- Abiertos y críticos ante los acontecimientos de la sociedad actual.

\section{A modo de conclusión}

La planificación y el diseño del proceso de enseñanzaaprendizaje es, en la actualidad, una de las competencias docentes más importantes de cara a los cambios que nos exige la filosofía del Espacio Europeo de Educación Superior. La concepción de la docencia destinada a favorecer el aprendizaje del alumno, de manera óptima, exige una adecuada planificación de la docencia. Con esto se incrementaría la probabilidad de que los estudiantes adquieran las competencias y objetivos previstos. Para ello, 
es necesario que el docente y los alumnos utilicen estrategias adecuadas que les permitan mejorar su aprendizaje y lograr los objetivos propuestos.

El tipo de profesionalización docente, que hoy se perfila, es la síntesis de varios elementos, entre los cuales se destacan: el carácter colectivo de la docencia, en comunidades de aprendizaje; el análisis sistemático y colaborativo de la propia práctica (reflexión sobre la acción desarrollada en el aula, y en la institución educativa propia, es decir, como individuo y también como parte de un colectivo); la actitud de perfeccionamiento permanente; el dominio de nuevas competencias docentes; la capacidad para tomar decisiones adecuadas. En suma, la formación inicial y permanente del profesorado son elementos indispensables para todos los proyectos de mejoramiento de la calidad de la educación.

\section{Referencias}

Buysse, V.; Sparkman, K. L., and Wesley, P. (2003). Communities of practice: Connecting what we know with what we do. Exceptional Children, 69(3), 263-277.

Cabero, J.; Villar, L. M. et al. (1998). "La utilización de las NN.TT. De la información y la comunicación en el desarrollo profesional docente: estudio cuantitativo". En M. Cebrián et al., Creación de materiales para la innovación educativa con las nuevas tecnologías (432446). Málaga: ICE Universidad de Málaga.

González, P. (Coord.) (1997). Psicología de los grupos. Teoría y aplicación. Madrid: Síntesis.

González, J., \& R. Wagenaar (2002). Tuning educational structures in Europe. Informe final fase 1. Bilbao: Universidad de Deusto.
Imbernon, F. (Coord.) (1993). La formación permanente del profesorado en los Países de la CEE. Barcelona: Horsori.

Instituto de Ciencias de la Educación (Universidad de Alicante). Orientaciones para la elaboración de guías docentes (2006). Alicante: Vicerrectorado de Planificación Estratégica y Calidad.

Lave, J., and Wenger, E. (1991). Situated learning: Legitimate peripheral participation. New York: Cambridge University Press.

Loepp, F. L. (1999). Models of curriculum integration. Journal of Technology Studies, 25(2), 21-25.

Marina, J.A. (2010). "Profesores para un mundo ultramoderno". En Cuadernos de Pedagogía, 304, 18-21.

Merma, G. (2008). “Competencias del profesorado para el uso de las tecnologías de la información y la comunicación en la enseñanza, en el marco del Espacio Europeo de Educación Superior". En R. Roig, et al., Investigación e innovación en el conocimiento educativo actual (317326). Alcoy: Marfil.

O'Sullivan, M., and Deglau, D. (2006). Chapter 7: Principles of professional development [Monograph]. Journal of Teaching in Physical Education, 25, 441-449.

Sarramona, J. (1991). Motivación y aprendizaje en el aula. Cómo enseñar a pensar. Madrid: Santillana.

Tejada, J. (1999). "El formador ante las NTIC: nuevos papeles y competencias profesionales". Comunicación y Pedagogía, 158, 17-26.

Vergnaud, G. (2001). "Problemas aditivos y multiplicativos". En M. C. Chamorro (Coord.) Dificultades del aprendizaje de las Matemáticas (191-193). Madrid: Ministerio de Educación, Cultura y Deporte.

Zabalza, M. A. (2003). Competencias docentes del profesorado universitario. Madrid: Narcea. 